\title{
SHORT CHARACTER SUMS WITH BEATTY SEQUENCES
}

\author{
William D. BANKs AND Igor E. ShPARLinski
}

\begin{abstract}
We estimate multiplicative character sums taken on the values of a nonhomogeneous Beatty sequence $\{\lfloor\alpha n+\beta\rfloor: n=1,2, \ldots\}$, where $\alpha, \beta \in \mathbb{R}$, and $\alpha$ is irrational. In particular, our bounds imply that for fixed $\alpha, \beta$ and a small real number $\varepsilon>0$, if $p$ is sufficiently large and $p^{1 / 3+\varepsilon} \leq N \leq p^{1 / 2+\varepsilon}$, then among the first $N$ elements of the Beatty sequence there are $N / 2+o(N)$ quadratic non-residues modulo $p$. If additional information about the Diophantine properties of $\alpha$ is available, then the error term $o(N)$ admits a sharper estimate. This work improves and extends several previous results due to M. Z. Garaev and S. N. Preobrazhenskiü.
\end{abstract}

\section{Introduction}

For fixed real numbers $\alpha$ and $\beta$, the associated non-homogeneous Beatty sequence is the sequence of integers defined by

$$
\mathcal{B}_{\alpha, \beta}=\{\lfloor\alpha n+\beta\rfloor: n=1,2,3, \ldots\} .
$$

Beatty sequences appear in a variety of apparently unrelated mathematical settings, and because of their versatility, the arithmetic properties of these sequences have been extensively explored in the literature; see, for example, $[1,3,6,12,13,16,17,23]$ and the references contained therein.

In this paper, as in [2], we study exponential sums of the form

$$
S_{p}(\alpha, \beta, \chi ; N)=\sum_{n \leq N} \chi(\lfloor\alpha n+\beta\rfloor),
$$

where $\alpha$ is irrational, and $\chi$ is a nontrivial multiplicative character modulo a prime number $p$. Our main result is Theorem 1 in Section 3 below. In particular, our bounds imply that for fixed $\alpha, \beta$ and a small real number $\varepsilon>0$, if $p$ is sufficiently large and $p^{1 / 3+\varepsilon} \leq N \leq p^{1 / 2+\varepsilon}$, then among the first $N$ elements of the Beatty sequence $\mathcal{B}_{\alpha, \beta}$, there are $N / 2+o(N)$ quadratic non-residues modulo $p$. In the case that $\alpha$ is not Liouville (which includes all algebraic irrationals and almost all real numbers), our results yield explicit bounds on the error term.

We remark that, if $N$ lies in the range $p^{1 / 2+\varepsilon} \leq N \leq p$, the results of [2] are stronger and more explicit, but they fail to provide a nontrivial bound when $N \leq p^{1 / 2}$. Hence, the results of this paper complement those of [2].

The method we use is similar to those used to estimate short double sums in modern proofs of the Burgess bound (see [9, Section 12.4]). Our underlying approach is based on some ideas from [10].

Various bounds on the size of the least quadratic non-residue modulo $p$ in the sequence $\mathcal{B}_{\alpha, 0}=\{\lfloor\alpha n\rfloor: n=1,2,3, \ldots\}$ have been obtained by Garaev [7] and

Received by the editors November 23, 2005.

2000 Mathematics Subject Classification. 11B50, 11L40, $11 \mathrm{~T} 24$. 
by Preobrazhenskiı $[18,19,20]$. For example, it has been shown in [7] that for any real number $\alpha>0$ and any prime $p$, there is a positive integer $n \leq p^{\left(1+e^{-1 / 2}\right) / 4+o(1)}$ such that $\lfloor\alpha n\rfloor$ is a quadratic non-residue modulo $p$. The bounds of $[18,19,20]$ are stronger than those of [7], but they require certain restrictions on the number $\alpha$.

Our results improve those of $[7,18,19,20]$ on the size of the least quadratic nonresidue. Moreover, the methods of those papers are much different from ours and cannot be applied to bound the character sums that we consider; in particular, they cannot be used to establish the uniform distribution of quadratic non-residues in Beatty sequences and do not apply to primitive roots at all.

\section{Notation}

Throughout the paper, the implied constants in the symbols $O$, « and $\gg$ may depend on the real numbers $\alpha$ and $\varepsilon$ but are absolute otherwise. We recall that the notations $U=O(V), U \ll V$, and $V \gg U$ are each equivalent to the assertion that the inequality $|U| \leq c V$ holds for some constant $c>0$. As usualy, $U \asymp V$ means $U \ll V \ll U$. We also use the symbol $o(1)$ to denote a function which tends to 0 and depends only on $\alpha$. It is important to note that our bounds are uniform with respect all of the involved parameters other than $\alpha$ and $\varepsilon$; in particular, our bounds are uniform with respect to $\beta$.

In what follows, the letters $k, m$, and $n$ (with or without subscripts) always denote non-negative integers unless indicated otherwise.

The notation $\|x\|$ is used to denote the distance from $x$ to the nearest integer; in other words,

$$
\|x\|=\min _{n \in \mathbb{Z}}|x-n| \quad(x \in \mathbb{R}) .
$$

We also use $\lfloor x\rfloor$ and $\{x\}$ to denote the greatest integer $\leq x$ and the fractional part of $x$, respectively.

The discrepancy $D$ of a sequence of $M$ (not necessarily distinct) real numbers $\gamma_{1}, \ldots, \gamma_{M} \in[0,1)$ is defined by

$$
D=\sup _{\mathcal{I} \subseteq[0,1)}\left|\frac{V(\mathcal{I}, M)}{M}-\right| \mathcal{I}||,
$$

where the supremum is taken over all subintervals $\mathcal{I}=(a, b)$ of the interval $[0,1)$, $V(\mathcal{I}, M)$ is the cardinality of the set $\left\{1 \leq m \leq M: \gamma_{m} \in \mathcal{I}\right\}$, and $|\mathcal{I}|=b-a$ is the length of $\mathcal{I}$.

Finally, we assume the reader is familiar with the basic properties of multiplicative characters (see, for example, [15]).

\section{Main result}

We use $D_{\alpha, \beta}(N)$ to denote the discrepancy of the sequence of fractional parts $\{\{\alpha n+\beta\}: n=1, \ldots, N\}$.

Theorem 1. There exists an absolute constant $c>0$ such that for any real numbers $\alpha, \beta, \varepsilon$ with $\alpha \neq 0$ and $0<\varepsilon<1 / 20$, a prime number $p$, a nontrivial multiplicative character $\chi(\bmod p)$, and an integer $p^{1 / 3+\varepsilon} \leq N \leq p^{1 / 2+\varepsilon}$, the upper bound

$$
\left|S_{p}(\alpha, \beta, \chi ; N)\right| \ll N p^{-c \varepsilon^{2}}+N D_{\alpha, \beta}(N)
$$


holds, where $c>0$ is an absolute constant, and the constant implied by $\ll$ depends only on $\alpha$ and $\varepsilon$.

Proof. We can assume that $\alpha>0$, since the case $\alpha<0$ is similar.

Let $K \leq N$ be a positive integer, and let $\Delta$ be a real number in the interval $(0,1]$. For every real number $\gamma$, let

$$
\begin{aligned}
& \mathcal{N}_{\gamma}=\{1 \leq n \leq N:\{\alpha n+\beta-\gamma\}<1-\Delta\} \\
& \mathcal{K}_{\gamma}=\{1 \leq k \leq K:\{\alpha k+\gamma\}<\Delta\}
\end{aligned}
$$

and put $\mathcal{N}_{\gamma}^{\mathbf{c}}=\{1,2, \ldots, N\} \backslash \mathcal{N}_{\gamma}$. From the definition (1), we immediately conclude that

$$
\# \mathcal{N}_{\gamma}^{\mathbf{c}}=N \Delta+O\left(N D_{\alpha, \beta}(N)\right) \text {. }
$$

As in the proof of [2, Theorem 4.1], we also have the lower bound

$$
\# \mathcal{K}_{\gamma} \geq 0.5 K \Delta
$$

for some choice of $\gamma \in(0,1]$. Fix $\gamma$ with this property, and put $\mathcal{N}=\mathcal{N}_{\gamma}, \mathcal{N}^{\mathbf{c}}=\mathcal{N}_{\gamma}^{\mathbf{c}}$ and $\mathcal{K}=\mathcal{K}_{\gamma}$.

We have for every $k \in \mathcal{K}:$

$$
\begin{aligned}
S_{p}(\alpha, \beta, \chi ; N) & =\sum_{n \leq N} \chi(\lfloor\alpha(n+k)+\beta\rfloor)+O(k) \\
& =\sum_{n \leq N} \chi(\lfloor\alpha(n+k)+\beta\rfloor)+O(K) \\
& =\sum_{n \in \mathcal{N}} \chi(\lfloor\alpha(n+k)+\beta\rfloor)+O\left(K+\# \mathcal{N}^{\mathbf{c}}\right) .
\end{aligned}
$$

Therefore,

$$
S_{p}(\alpha, \beta, \chi ; N)=\frac{W_{1}}{\# \mathcal{K}}+O\left(K+\# \mathcal{N}^{\mathbf{c}}\right),
$$

where

$$
W_{1}=\sum_{n \in \mathcal{N}} \sum_{k \in \mathcal{K}} \chi(\lfloor\alpha(n+k)+\beta\rfloor) .
$$

For any $n \in \mathcal{N}$ and $k \in \mathcal{K}$, we have

$$
\begin{aligned}
\lfloor\alpha(n+k)+\beta\rfloor & =\alpha(n+k)+\beta-\{\alpha(n+k)+\beta\} \\
& =(\alpha n+\beta-\gamma)+(\alpha k+\gamma)-\{\alpha n+\beta-\gamma\}-\{\alpha k+\gamma\} \\
& =\lfloor\alpha n+\beta-\gamma\rfloor+\lfloor\alpha k+\gamma\rfloor .
\end{aligned}
$$

We also remark that, since $N \leq p$, we have

$$
\#\{n \in \mathcal{N}:\lfloor\alpha n+\beta-\gamma\rfloor \equiv s \quad(\bmod p)\}=O(1)
$$


uniformly for all $s \in \mathbb{Z}$. Therefore, applying the Cauchy inequality, we derive that

$$
\begin{aligned}
\left|W_{1}\right|^{2} & \leq N \sum_{n \in \mathcal{N}}\left|\sum_{k \in \mathcal{K}} \chi(\lfloor\alpha n+\beta-\gamma\rfloor+\lfloor\alpha k+\gamma\rfloor)\right|^{2} \\
& \ll N \sum_{x=1}^{X}\left|\sum_{y \in \mathcal{Y}} \psi(y) \chi(x+y)\right|^{2},
\end{aligned}
$$

where $X=\lceil\alpha N\rceil, \mathcal{Y}$ is the set of values taken by $\lfloor\alpha k+\gamma\rfloor$ as $k$ varies over the set $\mathcal{K}$, and $\psi(y)$ is the multiplicity of each $y \in \mathcal{Y}$ (that is, the number of $k \in \mathcal{K}$ such that $\lfloor\alpha k+\gamma\rfloor=y)$. Therefore, we have

$$
\begin{aligned}
\left|W_{1}\right|^{2} & \ll N \sum_{y_{1}, y_{2} \in \mathcal{Y}} \psi\left(y_{1}\right) \psi\left(y_{2}\right)\left|\sum_{x=1}^{X} \chi\left(x+y_{1}\right) \bar{\chi}\left(x+y_{2}\right)\right| \\
& \ll N \sum_{y_{1}, y_{2} \in \mathcal{Y}}\left|\sum_{x=1}^{X} \chi\left(x+y_{1}\right) \bar{\chi}\left(x+y_{2}\right)\right|,
\end{aligned}
$$

where we have used the fact that $\psi(y)=O(1)$ for $y \in \mathcal{Y}$. Now, for any positive real numbers $U$ and $V$, we have

$$
\sum_{x=1}^{X} \chi\left(x+y_{1}\right) \bar{\chi}\left(x+y_{2}\right)=\sum_{x=1}^{X} \chi\left((x+u v)+y_{1}\right) \bar{\chi}\left((x+u v)+y_{2}\right)+O(U V)
$$

uniformly for $1 \leq u \leq U$ and $1 \leq v \leq V$; thus, if $\max \{U, V\}<p$, it follows that

$$
\begin{aligned}
& \sum_{x=1}^{X} \chi\left(x+y_{1}\right) \bar{\chi}\left(x+y_{2}\right) \\
& =\frac{1}{U V} \sum_{x=1}^{X} \sum_{1 \leq u \leq U} \sum_{1 \leq v \leq V} \chi\left(x+y_{1}+u v\right) \bar{\chi}\left(x+y_{2}+u v\right)+O(U V) \\
& =\frac{1}{U V} \sum_{x=1}^{X} \sum_{1 \leq u \leq U} \sum_{1 \leq v \leq V} \chi\left(\left(x+y_{1}\right) u^{-1}+v\right) \bar{\chi}\left(\left(x+y_{2}\right) u^{-1}+v\right)+O(U V),
\end{aligned}
$$

since $\chi(u) \bar{\chi}(u)=1$. Here, $u^{-1}$ denotes the inverse of $u$ in the multiplicative group modulo $p$. Inserting this expression into (5), we see that

$$
\left|W_{1}\right|^{2} \ll \frac{N}{U V} W_{2}+N Y^{2} U V
$$

where $Y=\# \mathcal{Y}$, and

$$
W_{2}=\sum_{x=1}^{X} \sum_{y_{1}, y_{2} \in \mathcal{Y}} \sum_{1 \leq u \leq U}\left|\sum_{1 \leq v \leq V} \chi\left(\left(x+y_{1}\right) u^{-1}+v\right) \bar{\chi}\left(\left(x+y_{2}\right) u^{-1}+v\right)\right| .
$$

Clearly, $W_{2}$ can be expressed in the form

$$
W_{2}=\sum_{x=1}^{X} \sum_{\lambda=1}^{p} \sum_{\mu=1}^{p} I(x, \lambda, \mu)\left|\sum_{1 \leq v \leq V} \chi(\lambda+v) \bar{\chi}(\mu+v)\right|,
$$


where $I(x, \lambda, \mu)$ denotes the number of solutions $\left(y_{1}, y_{2}, u\right)$, with $y_{1}, y_{2} \in \mathcal{Y}$ and $1 \leq$ $u \leq U$, to the system of congruences

$$
\left(x+y_{1}\right) u^{-1} \equiv \lambda \quad(\bmod p) \quad \text { and } \quad\left(x+y_{2}\right) u^{-1} \equiv \mu \quad(\bmod p) .
$$

Writing

$$
W_{2}=\sum_{x=1}^{X} \sum_{\lambda=1}^{p} \sum_{\mu=1}^{p} I(x, \lambda, \mu)^{(\nu-1) / \nu}\left(I(x, \lambda, \mu)^{2}\right)^{1 / 2 \nu}\left|\sum_{1 \leq v \leq V} \chi(\lambda+v) \bar{\chi}(\mu+v)\right|,
$$

and applying the Hölder inequality, we have

$$
W_{2}^{2 \nu} \leq\left(\sum_{x=1}^{X} \sum_{\lambda=1}^{p} \sum_{\mu=1}^{p} I(x, \lambda, \mu)\right)^{2 \nu-2}\left(\sum_{x=1}^{X} \sum_{\lambda=1}^{p} \sum_{\mu=1}^{p} I(x, \lambda, \mu)^{2}\right) X W_{3}
$$

for any integer $\nu \geq 1$, where

$$
W_{3}=\sum_{\lambda=1}^{p} \sum_{\mu=1}^{p}\left|\sum_{1 \leq v \leq V} \chi(\lambda+v) \bar{\chi}(\mu+v)\right|^{2 \nu} .
$$

It is easy to see that

$$
\sum_{x=1}^{X} \sum_{\lambda=1}^{p} \sum_{\mu=1}^{p} I(x, \lambda, \mu) \leq X Y^{2} U
$$

Also, for each fixed value of $x$, we have

$$
\sum_{\lambda=1}^{p} \sum_{\mu=1}^{p} I(x, \lambda, \mu)^{2}=T_{x}
$$

where $T_{x}$ is the number of solutions $\left(y_{1}, y_{2}, z_{1}, z_{2}, u, w\right)$, with $y_{1}, y_{2}, z_{1}, z_{2} \in \mathcal{Y}$ and $1 \leq u, w \leq U$, to the system of congruences

$$
\begin{aligned}
\left(x+y_{1}\right) u^{-1} & \equiv\left(x+z_{1}\right) w^{-1} \quad(\bmod p), \\
\left(x+y_{2}\right) u^{-1} & \equiv\left(x+z_{2}\right) w^{-1} \quad(\bmod p) .
\end{aligned}
$$

This system is clearly equivalent to the system

$$
\begin{array}{ll}
\left(x+y_{1}\right) w \equiv\left(x+z_{1}\right) u & (\bmod p), \\
\left(x+y_{2}\right) w \equiv\left(x+z_{2}\right) u & (\bmod p) .
\end{array}
$$

To proceed further, let us suppose that the bound

$$
\left(X+\max _{y \in \mathcal{Y}}\{y\}\right) U<p
$$

holds; below, we choose $U$ to satisfy this condition. Using (10), it follows that

$$
\max \left\{\left(x+y_{1}\right) w,\left(x+z_{1}\right) u,\left(x+y_{2}\right) w,\left(x+z_{2}\right) u\right\}<p,
$$

which implies that the congruences (9) are in fact equalities:

$$
\left(x+y_{1}\right) w=\left(x+z_{1}\right) u \quad \text { and } \quad\left(x+y_{2}\right) w=\left(x+z_{2}\right) u .
$$

Therefore, if $x, y_{1}, y_{2}$, and $w$ are fixed, then there are at most $\tau\left(\left(x+y_{1}\right) w\right)$ choices for each of $z_{1}$ and $u$ (where $\tau(s)$ denotes the number of positive divisors of an integer 
$s$ ), and after selecting $u$, the value of $z_{2}$ is determined uniquely. Using the well-known estimate $\tau(s)=s^{o(1)}$ (see [8, Theorem 317]), we conclude that

$$
\sum_{x=1}^{X} \sum_{\lambda=1}^{p} \sum_{\mu=1}^{p} I(x, \lambda, \mu)^{2}=\sum_{x=1}^{X} T_{x} \leq X Y^{2} U p^{o(1)} .
$$

Thus, from (7), (8) and (11), we derive the bound

$$
W_{2} \leq X Y^{2-1 / \nu} U^{1-1 / 2 \nu} p^{o(1)} W_{3}^{1 / 2 \nu} .
$$

Next, observe that

$$
\begin{aligned}
W_{3} & =\sum_{\lambda=1}^{p} \sum_{\mu=1}^{p}\left|\sum_{1 \leq v \leq V} \chi(\lambda+v) \bar{\chi}(\mu+v)\right|^{2 \nu} \\
& =\sum_{1 \leq v_{1}, \ldots, v_{2 \nu} \leq V}\left|\sum_{\lambda=1}^{p} \chi\left(\left(\lambda+v_{1}\right) \cdots\left(\lambda+v_{\nu}\right)\right) \bar{\chi}\left(\left(\lambda+v_{\nu+1}\right) \cdots\left(\lambda+v_{2 \nu}\right)\right)\right|^{2} .
\end{aligned}
$$

For the $O\left(V^{\nu}\right)$ vectors $\left(v_{1}, \ldots, v_{2 \nu}\right)$ for which the first $\nu$ components are some permutation of the last $\nu$ components, we use the trivial bound $O(p)$ on the inner sum; for the remaining vectors, we use the Weil bound $O\left(p^{1 / 2}\right)$ (see [15, Chapter 5]). Hence, choosing

we obtain the estimate

$$
V=p^{1 / \nu}
$$

$$
W_{3} \ll V^{\nu} p^{2}+V^{2 \nu} p \ll p^{3} .
$$

After substituting this bound into (12), we see that

$$
W_{2} \leq X Y^{2-1 / \nu} U^{1-1 / 2 \nu} p^{3 / 2 \nu+o(1)} .
$$

Applying this estimate to (6) and taking into account our choice of $V$ above, we have

$$
\left|W_{1}\right|^{2} \ll N X Y^{2-1 / \nu} U^{-1 / 2 \nu} p^{1 / 2 \nu+o(1)}+N Y^{2} U p^{1 / \nu}
$$

We now choose

$$
U=X p^{-1 / 2 \nu}
$$

which leads to the estimate

$$
\left|W_{1}\right| \ll N^{1 / 2} X^{1 / 2-1 / 4 \nu} Y^{1-1 / 2 \nu} p^{(2 \nu+1) / 8 \nu^{2}+o(1)}+N^{1 / 2} Y X^{1 / 2} p^{-3 / 4 \nu} .
$$

Now, since $Y=\# \mathcal{Y} \asymp \# \mathcal{K}$ and $X \ll N$, from (4) we derive that

$$
\left|S_{p}(\alpha, \beta, \chi ; N)\right| \ll N^{1-1 / 4 \nu}(\# \mathcal{K})^{-1 / 2 \nu} p^{(2 \nu+1) / 8 \nu^{2}+o(1)}+N p^{-3 / 4 \nu}+K+\# \mathcal{N}^{\mathbf{c}} .
$$

Finally, using the bounds (2) and (3) we have

$$
\begin{aligned}
& \left|S_{p}(\alpha, \beta, \chi ; N)\right| \\
& \quad \ll N^{1-1 / 4 \nu}(K \Delta)^{-1 / 2 \nu} p^{(2 \nu+1) / 8 \nu^{2}+o(1)}+N p^{-3 / 4 \nu}+K+N \Delta+N D_{\alpha, \beta}(N) .
\end{aligned}
$$

We now choose

$$
\Delta=N^{-3 /(4 \nu+4)} p^{(2 \nu+1) /\left(8 \nu^{2}+8 \nu\right)} \quad \text { and } \quad K=\lceil N \Delta\rceil,
$$

obtaining the bound

$$
\left|S_{p}(\alpha, \beta, \chi ; N)\right| \ll N \Delta+N p^{-3 / 4 \nu}+N D_{\alpha, \beta}(N) .
$$


Taking $\nu=\left\lceil(5 \varepsilon)^{-1}\right\rceil$ and bearing in mind that $N \geq p^{1 / 3+\varepsilon}$, one sees that

$$
\Delta \leq p^{-5 \varepsilon^{2} /(8+40 \varepsilon)} \quad \text { and } \quad p^{-3 / 4 \nu} \leq p^{-15 \varepsilon /(4+20 \varepsilon)} .
$$

In particular, $\Delta \in(0,1]$ as required, and inserting these inequalities into (13), we obtain the bound stated in the theorem.

To finish the proof, it remains to check that the bound (10) holds with the parameter choices made above. Indeed,

$$
\left(X+\max _{y \in \mathcal{Y}}\{y\}\right) U \ll N U=N X p^{-1 / 2 \nu} \ll N^{2} p^{-1 / 2 \nu} .
$$

Since $\nu=\left\lceil(5 \varepsilon)^{-1}\right\rceil$ and $N \leq p^{1 / 2+\varepsilon}$, it follows that

$$
\left(X+\max _{y \in \mathcal{Y}}\{y\}\right) U \ll p^{\left(2+9 \varepsilon+20 \varepsilon^{2}\right) /(2+10 \varepsilon)} .
$$

Since $\varepsilon<1 / 20$,

$$
\frac{2+9 \varepsilon+20 \varepsilon^{2}}{2+10 \varepsilon}<1
$$

and therefore (10) holds once $p$ is sufficiently large.

\section{Applications}

Since $D_{\alpha, \beta}(N)=o(1)$ for every irrational number $\alpha$ (see [14, Example 2.1, Chapter 1]), we have:

Corollary 1. If $\alpha$ is irrational, then under the conditions of Theorem 1, the following bound holds:

$$
S_{p}(\alpha, \beta, \chi ; N)=o(N) .
$$

If more information about the Diophantine properties of $\alpha$ is available, one can derive a more precise upper bound.

For an irrational number $\alpha$, we define its type $\tau$ by the relation

$$
\tau=\sup \left\{\vartheta \in \mathbb{R}: \liminf _{q \rightarrow \infty, q \in \mathbb{Z}^{+}} q^{\vartheta}\|\alpha q\|=0\right\} .
$$

The celebrated theorems of Khinchin [11] and of Roth [21] assert that $\tau=1$ for almost all real (in the sense of the Lebesgue measure) and all irrational algebraic numbers $\alpha$, respectively, while the Dirichlet theorem asserts that $\tau \geq 1$ for every irrational $\alpha$; see also $[4,22]$. We remark that the number $\mu=\tau+1$ is called the irrationality measure of $\alpha$, or the Liouville-Roth constant.

By [14, Theorem 3.2, Chapter 2], we have $D_{\alpha, \beta}(N) \leq N^{-1 / \tau+o(1)}$ for any irrational $\alpha$ of type $\tau<\infty$. Therefore, we have:

Corollary 2. If $\alpha$ is irrational of type $\tau<\infty$, then under the conditions of Theorem 1, the following bound holds:

$$
\left|S_{p}(\alpha, \beta, \chi ; N)\right| \ll N p^{-c \varepsilon^{2}}+N^{1-1 / \tau+o(1)} .
$$

Let $N_{\alpha}(p)$ be the smallest integer $n \geq 1$ for which $\lfloor\alpha n\rfloor$ is a quadratic non-residue modulo $p$. The following bound improves those of $[7,18,19,20]$. 
Corollary 3. For any real numbers $\alpha, \varepsilon$ with $\alpha \neq 0$ and $\varepsilon>0$, the following bound holds for all primes $p$ :

$$
N_{\alpha}(p) \ll p^{1 / 3+\varepsilon} .
$$

Proof. If $\alpha$ is irrational the result follows immediately from Corollary 1. If $\alpha=r / s$ is rational with relatively prime integers $r$ and $s>0$, then

- if $r$ is a quadratic non-residue modulo $p, N_{\alpha}(p) \leq s=O(1)$,

- if $r$ is a quadratic residue modulo $p, N_{\alpha}(p) \leq s N(p)=O\left(p^{1 /(4 \sqrt{e})}\right)$, where $N(p)$ is the smallest quadratic non-residue modulo $p$ for which the Burgess bound applies (see [5, 9]).

This concludes the proof.

Finally, we remark that with Theorem 1 one can easily derive in a routine fashion many results about the uniformity of distribution of higher degree residues and nonresidues, and also of primitive roots, in Beatty sequences.

\section{Acknowledgements}

This work was done during a pleasant visit by W. B. to Macquarie University; the support and hospitality of this institution are gratefully acknowledged. During the preparation of this paper, I. S. was supported in part by ARC grant DP0556431.

\section{References}

[1] A. G. Abercrombie, Beatty sequences and multiplicative number theory, Acta Arith. 70 (1995), no. 3, 195-207.

[2] W. Banks and I. E. Shparlinski, Non-residues and primitive roots in Beatty sequences, to appear in Bull. Austral. Math. Soc.

[3] A. V. Begunts, An analogue of the Dirichlet divisor problem, Moscow Univ. Math. Bull. 59 (2004), no. 6, 37-41.

[4] Y. Bugeaud, Approximation by algebraic numbers, Cambridge University Press, Cambridge, 2004.

[5] D. A. Burgess, On character sums and primitive roots, Proc. London Math. Soc. 12 (1962) 179-192.

[6] A. S. Fraenkel and R. Holzman, Gap problems for integer part and fractional part sequences, J. Number Theory 50 (1995), no. 1, 66-86.

[7] M. Z. Garaev, A note on the least quadratic non-residue of the integer-sequences, Bull. Austral. Math. Soc. 68 (2003), no. 1, 1-11.

[8] G. H. Hardy and E. M. Wright, An introduction to the theory of numbers, Oxford Univ. Press, Oxford, 1979.

[9] H. Iwaniec and E. Kowalski, Analytic number theory, Amer. Math. Soc., Providence, RI, 2004.

[10] A. A. Karatsuba, The distribution of values of Dirichlet characters on additive sequences, Doklady Acad. Sci. USSR 319 (1991), 543-545.

[11] A. Ya. Khinchin, Zur metrischen Theorie der diophantischen Approximationen, Math. Z. 24 (1926), no. 4, 706-714.

[12] T. Komatsu, A certain power series associated with a Beatty sequence, Acta Arith. 76 (1996), no. 2, 109-129.

[13] (1995), no. 2, 387-406.

[14] L. Kuipers and H. Niederreiter, Uniform distribution of sequences, Wiley-Interscience, New York-London-Sydney, 1974.

[15] R. Lidl and H. Niederreiter, Finite fields, Cambridge University Press, Cambridge, 1997. 
[16] G. S. Lü and W. G. Zhai, The divisor problem for the Beatty sequences, Acta Math. Sinica 47 (2004) 1213-1216.

[17] K. O'Bryant, A generating function technique for Beatty sequences and other step sequences, J. Number Theory 94 (2002), no. 2, 299-319.

[18] S. N. Preobrazhenskiǔ, On the least quadratic non-residue in an arithmetic sequence, Moscow Univ. Math. Bull. 56 (2001), no. 1, 44-46.

[19] _ On power non-residues modulo a prime number in a special integer sequence, Moscow Univ. Math. Bull. 56 (2001), no. 4, 41-42.

[20] _ On the least power non-residue in an integer sequence, Moscow Univ. Math. Bull. 59 (2004), no. 1, 33-35.

[21] K. F. Roth, Rational approximations to algebraic numbers, Mathematika 2 (1955), 1-20; 'Corrigendum', Mathematika 2 (1955), 168.

[22] W. M. Schmidt, Diophantine approximation, Springer-Verlag, Berlin, 1980.

[23] R. Tijdeman, Exact covers of balanced sequences and Fraenkel's conjecture, Algebraic number theory and Diophantine analysis (Graz, 1998), pp. 467-483, de Gruyter, Berlin, 2000.

Department of Mathematics, University of Missouri, Columbia, MO 65211

E-mail address: bbanks@math.missouri.edu

Department of Computing,Macquarie University, Sydney, NSW 2109, Australia

E-mail address: igor@ics.mq.edu.au 